\title{
Förderung bildungssprachlicher Lexik im Jahrgang 5 - Empirische Evaluation eines integrativen Förderkonzepts
}

\author{
Britta-Katharina Ehrig • Nicole Marx • Lena Neumann
}

Online publiziert: 18. Mai 2020

(C) Der/die Autor(en) 2020

Zusammenfassung Auch wenn das Beherrschen altersangemessener bildungssprachlicher Lexik einen wesentlichen Faktor schulischen Erfolgs darstellt, werden entsprechende Kompetenzen in deutschen Schulen bislang kaum ausreichend vermittelt. Entsprechend wurde im Rahmen der hier vorgestellten Studie basierend auf der Robust Vocabulary Instruction (RVI, Beck et al. 2002) eine achtwöchige Intervention zur integrierten Förderung bildungssprachlicher Lexik für den fünften Jahrgang eines Gymnasiums entwickelt.

Die quasi-experimentelle Pilotstudie zeigte bei einem Prä-Posttest eine signifikante Verbesserung der Interventionsgruppe im Vergleich zur Kontrollgruppe. Darüber hinaus zeigten ANOVAs mit Messwiederholung, dass sich zwar beide Gruppen über den relativ kurzen Zeitraum verbesserten, die Interventionsgruppe sich jedoch signifikant stärker verbesserte. Zudem zeigte sich, dass eine Verbesserung nur in Bezug auf explizit trainierte oder zumindest von der Lehrkraft angesprochene Lexeme nachweisbar war. Ein rein implizites Wortschatzlernen nach nur rezeptiver Begegnung erwies sich nicht als erfolgreich.

\footnotetext{
M. Ed. B.-K. Ehrig $(\bowtie)$

Afdeling Master Leraar Duits, NHL Stenden Hogeschool, Eemsgolaan 17, 9727DW Groningen,

Niederlande

E-Mail: britta.ehrig@nhlstenden.com

Universität Köln, Köln, Deutschland

Prof. Dr. N. Marx

Institut für Deutsche Sprache und Literatur II, Mercator-Institut für Sprachförderung und Deutsch als Zweitsprache, Universität zu Köln, Albertus-Magnus-Platz, 50923 Köln, Deutschland

E-Mail: n.marx@uni-koeln.de

M. Ed. L. Neumann

Gymnasium Horn, Vorkampsweg 5, 28359 Bremen, Deutschland

E-Mail: lena.neumann@schule.bremen.de
} 
Schlüsselwörter Bildungssprache · Integrative Sprachförderung ·

Interventionsstudie $\cdot$ Sekundarstufe I - Wortschatz

\title{
Increasing academic vocabulary understanding and use in grade 5 - an intervention study
}

\begin{abstract}
Age-appropriate knowledge of academic vocabulary has been purported to be essential for success in school. Considering the importance of academic vocabulary and the conditions under which it is learned (extensive reading and reflection of texts in relevant genres and school subjects), it is surprising that to date, relatively little attention has been afforded its support in German schools. The reported study thus first developed an eight-week integrated classroom vocabulary support programme for fifth grade based on Robust Vocabulary Instruction (Beck et al. 2002). It then compared the results of an intervention (RVI) group with those of three control groups in a pre-/posttest design. Results of the repeated-measures ANOVAs indicated that, while both groups showed significant improvement, even within the relatively short two-month study, the RVI group performed significantly better. Further analyses showed that the gain was, however, only detectable for intensively trained or explicitly addressed vocabulary. Implicit vocabulary learning solely through encounter during class did not prove conducive to vocabulary learning.
\end{abstract}

Keywords Academic language · Integrated language support · Intervention study · Secondary school $\cdot$ Vocabulary

\section{Einleitung}

Sprachliche Kompetenzen und insbesondere bildungssprachliche Kompetenzen gelten als grundlegend für den schulischen Erfolg (z. B. Gogolin 2006; Feilke 2012; Schleppegrell 2012; Townsend et al. 2012), ermöglichen sie doch ,die Partizipation an Unterrichtsgesprächen, das Verstehen und Verarbeiten von Texten sowie die Erstellung eigener fachlicher Texte - und zwar über alle Fächer hinweg“ (Quasthoff 2009, S. 84f.). Besonders viel Aufmerksamkeit wird dabei dem Wortschatz als „Schaltstelle“ des schulischen Lernens gewidmet (Steinhoff 2009; Ulrich 2011, S. 9f.), da ein wesentlicher Anteil des Wissenserwerbs der Lernenden über das Wortschatzinventar erfolgt. Dies liegt insbesondere an der Präsentation fachlicher Inhalte in schriftlichen Texten und damit verbunden an der Tatsache, dass der Wortschatzumfang ein starker Prädikator für Leseverstehen ist (s. die Forschungszusammenfassung in Mckee 2012). Zudem bedingen sich Wortschatzerwerb und das Verständnis komplexer Konzepte gegenseitig (vgl. Ahrenholz 2010, S. 18f.).

Wenngleich einige Lernende durch einen impliziten Zugang den bildungsrelevanten Wortschatz erwerben, was v.a. durch eine häufige Lexemkonfrontation in unterschiedlichen Kontexten ermöglicht wird (vgl. Pigada und Schmitt 2006; Behrens 2009), ist dieser Zugang nicht für alle SchülerInnen zu erwarten (vgl. u.a. Steinig 2016, S. 91 f.). Insgesamt scheint der Erwerb bildungssprachlicher Lexik für fast alle Lernenden eine Herausforderung darzustellen (vgl. Feilke 2012, S. 8). So 
zeigen beispielsweise die Ergebnisse der DESI-Studie, dass Wortschatzkenntnisse bezogen auf drei Zielbereiche (A: die 2000 am häufigsten auftretenden Wörter des Deutschen; B: häufige Konkreta/Abstrakta; C: seltene Fach- und Fremdwörter) von fast $40 \%$ der SchülerInnen des 9. Jahrgangs unterhalb des altersangemessenen Kompetenzniveaus liegen (vgl. Willenberg 2008, S. 76).

Angesichts der Bedeutung bildungssprachlicher Lexik für den Bildungserfolg und ihres erschwerten Erwerbs obliegt es Bildungsinstitutionen, sie gezielt zu fördern. Gerade hier zeigen sich Lücken in der Unterrichtspraxis. Dies ist nicht nur der Unklarheit des Konstrukts in Bezug auf Funktionen, Merkmale und erwartete Kompetenzen im Entwicklungsverlauf geschuldet. Auch fehlen weitgehend empirisch geprüfte Förderkonzepte.

Der vorliegende Beitrag widmet sich diesen Desideraten. Erstens werden Operationalisierungen von ,bildungssprachlichem Wortschatz“ diskutiert. Zweitens wird ein integratives Förderkonzept vorgestellt, das den Ausbau eines entsprechenden Wortschatzes unterstützt. Und drittens wird dieses Konzept in einer explorativen Intervention in der fünften Jahrgangsstufe empirisch geprüft, wobei hier lediglich die quantitativen Daten aus Wortschatzprä- und -posttests präsentiert werden. Ziel ist es, Hinweise auf eine in der Schule realisierbare Vermittlung bildungssprachlichen Wortschatzes zu entwickeln.

\section{Bildungssprachlicher Wortschatz in der Schule}

Der Begriff „Bildungssprache“ hat in den letzten Jahren zwar hohe Konjunktur erfahren, bleibt aber - vielleicht auch gerade wegen seiner Präsenz in unterschiedlichen Kontexten - recht diffus (s. exemplarisch die Diskussion in Steinhoff 2019). Dies hängt auch damit zusammen, dass eine Abgrenzung von anderen Ausprägungen von Sprache (in vielen Konzepten als Register dargelegt) größtenteils noch nicht erfolgt ist (s.a. Snow und Uccelli 2009, S. 121; Morek und Heller 2012, S. 73). Allen Konzepten gemeinsam ist der Ansatz, dass Wortschatz eine zentrale Komponente von Bildungssprache bildet und im institutionellen Kontext durch die Auseinandersetzung mit konzeptionell schriftsprachlichem Material (vgl. Koch und Österreicher 1985) gefördert werden kann.

Im deutschsprachigen Raum sind bislang unterschiedliche Vorgehensweisen zur Operationalisierung des bildungssprachlichen Wortschatzes leitend gewesen. Ein populärer Ansatz setzt sich zum Ziel, Merkmalslisten aus unterschiedlichen Quellen zu entwickeln (Korpusanalysen, Experteneinschätzungen, Häufigkeitsanalysen, Übersetzungen von englischen Wortlisten). So werden Merkmale wie nichtfachliche Fremdwörter (z. B. Definition, Ortner 2009, S. 2223), unpersönliche Ausdrücke (z. B. man, Gogolin et al. 2007, S. 42) oder Funktionsverbgefüge (z. B. zur Diskussion stellen, Kurtz 2012a, S. 244) genannt. Solche Wortschatz- bzw. Kriterienlisten sind allerdings nicht unproblematisch, verführen sie doch zu Vereinfachungen, Übergeneralisierungen oder sogar zur Missachtung der zu erfüllenden Funktionen; zudem erweisen sie sich für das Deutsche als wenig zielführend. Und schließlich können die auf ihnen stehenden Items auch in alltagssprachlichen Kontexten frequent sein (s.u.a. Stahns 2016; Marx 2018; Steinhoff 2019). 
Vielversprechend scheinen dagegen Ansätze, die nach möglichen Funktionen von Bildungssprache fragen. Ist Bildungssprache ,funktional mit Anforderungen der Wissensdarstellung und Wissenskommunikation verbunden" (Feilke 2012, S. 10), stehen die Wissensindizierung (sozialsymbolische Funktion), der Wissenserwerb (epistemische Funktion) und die Wissensverständigung (Kommunikation über akademische Inhalte) im Vordergrund (vgl. Morek und Heller 2012). Dass alle Funktionen gleichzeitig eine Rolle spielen, ist anhand von Beispielen wie speziell (im Kontrast zu besonders) oder unmittelbar (anstatt sofort) gut zu erkennen. So kann die Verwendung der Begriffe speziell und unmittelbar eine (1) sozialsymbolische Funktion erfüllen, indem es einen Sprechenden als einer (höheren) Bildungsklasse zugehörig ausweist, (2) eine epistemische Funktion erfüllen, indem die Begriffe zum Aufbau eines Verständnisses und zur Aneignung komplexer Zusammenhänge dienen und (3) eine kommunikative Funktion erfüllen, indem die Begriffe zur Explikation im bildungssprachlichen Dialog eingesetzt werden. Solche bildungssprachlichen Begriffe, für die es häufig alltagssprachliche Synonyme gibt, sind vorwiegend im schriftsprachlichen Register vorzufinden.

Insbesondere in Bezug auf die Funktion der Wissensindizierung hat sich ein Förderkonzept von Beck et al. (2002), das für den deutschsprachigen Raum von Kurtz (2012a) aufgenommen, adaptiert und didaktisch umgesetzt wurde, als gewinnbringend erwiesen. Anders als die oben beschriebenen Zugänge zu Bildungssprache bezieht sich dieser Ansatz auf Bildungssprache als Register. Da bei diesem eine Entwicklungsperspektive eingenommen und v. a. semantische und frequenzbasierte Merkmale zugrunde gelegt werden, können Kriterien formuliert werden, die von in der Praxis tätigen Lehrkräften leicht umzusetzen sind. Bildungssprachliche Wortschatzeinheiten erfüllen zwar noch die im funktionalen Ansatz vorgelegten Funktionen, ihnen werden aber ,prototypische“ Merkmale zugeschrieben, die besonders häufig auf bildungssprachlichen Wortschatz zutreffen. Sie seien (1) in schriftnahen Kontexten hochfrequent, im alltagssprachlichen Gebrauch hingegen weniger vertreten, (2) domänenunspezifisch und somit interdisziplinär und/oder (3) konzeptuell reich und dementsprechend gut vernetzbar. Sie können auch (4) eine spezifische Funktion im Text übernehmen, (5) metaphorisch oder idiomatisch verwendet werden oder (6) für die grammatik- oder textbezogene Spracharbeit durch ihre Komplexität sowie ihr häufiges Auftreten von übergeordneter Relevanz sein (adaptiert aus Beck et al. 2008, S. 7 ff.; Kurtz 2012b, S. 78f.). Damit wird für Lehrende sprachlicher und nichtsprachlicher Fächer eine praxistaugliche Operationalisierung von Bildungssprache vorgenommen.

Aufgrund einer angestrebten Praxisnähe wurde in der vorliegenden Studie erstens entschieden, bei der Wahl der zu behandelnden Einheiten von der Musterhaftigkeit für bildungssprachliche Funktionen (vgl. Feilke 2012) in genrespezifischem und fachübergreifendem Gebrauch auszugehen. Zweitens wird zur Unterstützung bei der konkreten Auswahl auf die o.g. funktionalen Kriterien von Beck et al. (2008) sowie Kurtz (2012b) rekurriert, anstatt sie aus strukturellen (grammatischen oder morphologischen) Merkmalen abzuleiten. Die Sinnhaftigkeit des Vorgehens bestätigte sich in den Pilotierungen, da die involvierten LehrerInnen damit zielgerichteter und begründeter weitere relevante Einheiten identifizieren konnten. 


\section{Förderung bildungssprachlichen Wortschatzes in der Schule}

Bislang wurden im deutschsprachigen Raum wenige Konzepte zur Förderung bildungssprachlichen Wortschatzes von SchülerInnen in den Sekundarstufen entwickelt (für die Grundschule vgl. Kurtz 2012b; Heppt 2016). Ein empirisch überprüftes Förderkonzept für SekundarschülerInnen steht somit noch aus. Um diese Lücke zu schließen, wurde auf Grundlage bereits erprobter Konzepte und inhärenter normativer Überlegungen ein Förderkonzept für den 5. Jahrgang entwickelt und an einem Bremer Gymnasium erprobt.

\subsection{Robust Vocabulary Instruction}

Die „Robust Vocabulary Instruction“ (RVI) von Beck et al. (2002) wurde bereits in den 1980er Jahren für den angloamerikanischen Raum entwickelt und seitdem mehrfach empirisch begleitet. Das Konzept folgt einem fächerübergreifenden, integrativen Ansatz. Ziel ist dabei der Aufbau eines ,robusten“ Bildungswortschatzes aller SchülerInnen. Entsprechend gängiger kognitionswissenschaftlicher Erkenntnisse wird eine von der Schriftlichkeit ausgehende Förderung aufgebaut, die dann von der schriftlichen über die mündliche Rezeption zur schriftlichen und dann mündlichen Produktion führt (vgl. hierzu auch Steinig 2016, S. 218; Steinhoff et al. 2019).

Beim Förderkonzept liegt der Fokus weniger auf der rezeptiven Einbindung einer möglichst hohen Anzahl an Lexemen, sondern auf der reichhaltigen Darstellung einzelner Lexeme in ihren verschiedenen morphologischen und semantischen Ausprägungen. Ausgewählte Wortschatzeinheiten (ca. zehn pro Woche) sollen in verschiedenen fachlichen Kontexten vorkommen oder als Modell dienlich sein. Durch ein breites Spektrum aktivierender Wortschatzaufgaben und das regelmäßige Aufgreifen der fokussierten Lexeme während des Unterrichts sollen die Zieleinheiten vielfältig vernetzt werden. Diese Überlegungen zeigen Ähnlichkeiten mit den aus der Fremdsprachendidaktik stammenden Ansätzen des ,impliziten“ und ,expliziten" Wortschatzlernens, wobei explizites Wortschatzlernen ein bewusstes und absichtliches Formen von Bedeutungen bezeichnet und implizites Wortschatzlernen von einer unbewussten Wahrnehmung durch wiederholten sprachlichen Input ausgeht (z. B. Tschirner 2010, S. 242). Im Rahmen des Förderkonzepts werden beide Ansätze miteinander verbunden.

Die Wortschatzarbeit im Unterricht erfolgt themenintegrativ, was zu einer erhöhten Lernermotivation, einer besseren Auffassung semantischer Nuancen einzelner Lexeme sowie zu einer stärkeren konzeptuellen Verknüpfung trainierter Einheiten mit fachlichen Inhalten führen soll (vgl. Kurtz 2012b, S. 79 f.). Durch die variierende aktive Einbindung der Zieleinheiten in den Unterricht wird deren Vernetzung in unterschiedlichen syntagmatischen und paradigmatischen Beziehungen angestrebt (vgl. Kurtz 2012b, S. 72, 81).

Im Rahmen verschiedener Studien mit Vor- und Grundschulkindern wurde die Effektivität des RVI nachgewiesen (vgl. Beck et al. 1982; McKeown et al. 1985). Dabei hängt die Intensität der Förderung direkt mit dem Wortschatzzuwachs zusammen, ungeachtet des geschätzten Schwierigkeitsgrads der Lexeme (vgl. Beck et al. 2002). 
Mit Kurtz (2012b) liegt eine Adaption für die dritte und vierte Jahrgangsstufe im deutschsprachigen Raum vor. Obwohl in einer neunmonatigen Interventionsstudie beim direkten Vergleich von Interventions- und Kontrollgruppe zunächst keine positiven Effekte dieses Wortschatztrainings nachgewiesen wurden, ergab eine detaillierte Analyse ein differenzierteres Bild für die einzelnen Interventionsgruppen: Während sich die Interventionsklassen mit wenig erprobten Förderlehrkräften nicht von der Kontrollgruppe unterschieden, konnte für Klassen mit erfahreneren Förderlehrkräften ein signifikanter Unterschied zwischen Kontroll- und Interventionsgruppe nachgewiesen werden (vgl. Kurtz 2012c, S. $184 \mathrm{ff}$.). Die Fördermaßnahme war also erfolgreich, allerdings nur, wenn die involvierten Lehrkräfte bereits zuvor die Gelegenheit hatten, RVI in den Unterricht einzubinden. Dies führte in der vorliegenden Studie zur Entscheidung, dass eine geschulte Masterstudentin die Lehrkräfte beim Einsatz des RVI unterstützte.

\subsection{Anpassung des Förderkonzepts für die 5. Klasse}

Basierend auf dem Konzept des RVI wurde ein Wortschatzförderkonzept für die fünfte Jahrgangsstufe eines Gymnasiums, das Wortschatzdefizite seiner SchülerInnen beobachtete und Interesse an einer Kooperation zeigte, entwickelt. Dieses Training wurde nach Ablauf des hier berichteten Projektes von der Schule eigenständig weitergeführt und ist noch heute fächerübergreifend und integrativ im Regelunterricht verankert. Der Fokus des Trainings liegt auf dem expliziten und intensiven Erwerb ausgewählter Wortschatzeinheiten, um deren dauerhafte Überführung in den produktiven Wortschatz zu ermöglichen (s. a. Ulrich 2011, S. 20).

Bei der Auswahl der Zieleinheiten werden einerseits die von Beck et al. (2008, S. 7 ff.) und Kurtz (2012b, S. 78 f.) aufgestellten Kriterien berücksichtigt. Andererseits wurden auch solche Redemittel herangezogen, die in bildungssprachlichen Texten die Funktionen der Explizierung, Verdichtung, Verallgemeinerung oder Diskussion (vgl. Feilke 2012) erfüllen. Die Einheiten werden in Vorbereitung auf die Unterrichtsstunde oder spontan im Unterrichtsgeschehen ausgewählt und sollen entweder den meisten SchülerInnen unbekannt oder nur in bestimmtem Umfeld bekannt sein, um eine Bedeutungserweiterung bzw. -vertiefung zu ermöglichen. Der erste Kontakt mit den Lexemen erfolgt stets textuell eingebettet im Kontext der aktuellen Unterrichtsmaterialien mindestens eines Faches. Das erlaubt erstens die sinnvolle Integration in den Regelunterricht und zweitens das Festigen mindestens einer von ggf. vielen Bedeutungsfacetten. Insgesamt werden ca. 20 Lexeme in jedem zweiwöchigen Trainingszyklus eingeführt.

Das Vorgehen im Unterricht verknüpft im Wesentlichen drei Komponenten: ein Wortschatzplakat, eine Wortschatzmappe und eine Auswahl an Wortschatzübungen. Das zunächst leere Wortschatzplakat wird an einem für alle SchülerInnen gut einsehbaren Ort im Klassenzimmer positioniert und alle zwei Wochen von der Klassenleitung erneuert. Während des Trainingszyklus wird das Plakat fortwährend im Unterricht um die zu fokussierenden Lexeme erweitert. Die Wortschatzeinheiten können von den Lehrkräften entweder spontan in den Regelunterricht integriert oder explizit aufgegriffen werden. Die SchülerInnen begegnen den Lexemen in unterschiedlichen fachlichen wie auch sprachlichen Kontexten und erweitern auf diese 
Weise sukzessive deren Bedeutungszuschreibung. Das Plakat dient nicht nur als Erinnerungsstütze, sondern auch als niedrigschwellige Kommunikationsmöglichkeit zwischen den Fachlehrkräften: Auch wenn die Lehrkräfte unterschiedlicher Fächer sich nicht zum Zielwortschatz austauschen, sind die Trainingseinheiten direkt dem Plakat zu entnehmen. Nach Ablauf eines zweiwöchigen Trainingszyklus werden die Lexeme dauerhaft sichtbar im Klassenraum fixiert, bspw. in einem (alphabetisch organisierten) Wortschatzzug, einer Schatzkiste oder einem Wörterbaum. So bleiben sie allen Beteiligten präsent und können weiterhin regelmäßig in Form verschiedener Übungen wie Reizwortgeschichten oder Tabu in den Unterricht integriert werden.

Die zweite Säule bildet die Wortschatzmappe. In dieser ist für jeden Buchstaben des Alphabets eine Seite reserviert. Jede Zieleinheit wird hier eingetragen und durch eine Definition und ein Beispiel ergänzt. Definitionen werden gemeinsam im Plenum diskutiert und auf diese Weise ggf. korrigiert oder erweitert. Die Wortschatzmappe wird in allen Fächern genutzt und soll stets griffbereit auf dem Tisch liegen, um einen Gewöhnungseffekt zu erzielen. Neben den zu trainierenden Lexemen werden die SchülerInnen dazu angeregt, weitere, ihnen unbekannte Redemittel in ihre Mappen aufzunehmen. Die Arbeit mit der Wortschatzmappe bildet v.a. zu Beginn den zeitintensivsten Teil des Trainings, bietet den SchülerInnen dafür aber die Möglichkeit, sich mit im Alltag niedrigfrequenten, für den Bildungsweg zentralen Lexemen intensiv auseinanderzusetzen. Zugleich fungiert die Mappe als Dokumentation.

Bedeutungsaushandlung und -vertiefung findet schließlich v. a. durch den Einsatz unterschiedlicher Wortschatzübungen statt, die von der kontextgebundenen Begegnung mit neuen lexikalischen Einheiten in den regulären Lernmaterialien hin zu deren Abstraktion und produktiven, angemessenen Verwendung führen sollen. Gezielte Übungen dienen dabei der Förderung einer vertieften Verarbeitung sowie dem Erlernen der Restriktionen für deren funktionalen Gebrauch. Sie basieren größtenteils auf bekannten Wortschatzübungen und haben eine zeitliche Dauer von zwei Minuten bis zu einer Unterrichtsstunde, wobei der Großteil aller Übungen ca. fünf Minuten in Anspruch nimmt. Zudem werden einige Übungen für die Arbeit zu Hause empfohlen. Sie sind somit gut in den Regelunterricht zu integrieren, beanspruchen wenig Zeit im regulären Unterrichtsgeschehen und bieten unterschiedlichste Erweiterungsund Verfestigungsgelegenheiten an. Sie werden entweder spontan durchgeführt, z. B. bei inhaltlichen (neues Thema etc.) oder sozialen Brüchen im Unterrichtsgeschehen (Unruhe der SchülerInnen) oder geplant, z. B. als Abschluss einer Unterrichtseinheit.

Zentral für den Erfolg des Programms sind drei weitere Aspekte, die im vorliegenden Projekt durchgeführt wurden. Erstens fand eine einleitende Lehrerfortbildung statt, in der gemeinsam mit den Sprach- und Sachfachlehrkräften die Prinzipien des RVI erarbeitet, erste Zieleinheiten begründet ausgewählt und Übungen erklärt und erprobt wurden. Die Lehrkräfte erhielten zudem eine Aktivitätenmappe mit Wortschatzübungen. Da aus organisatorischen Gründen seitens der Schule keine weiteren Fortbildungen während der Projektphase durchgeführt werden konnten, war der dritte Aspekt besonders relevant: Eine geschulte Masterstudentin unterstützte für zehn Unterrichtsstunden pro Woche die involvierten Lehrkräfte bei der Auswahl passender zeitlicher Sequenzen und Wortschatzeinheiten und führte anteilig modellhafte Ziellexeme in der Klasse ein. Diese Studentin führte sowohl allein als auch gemeinsam mit den Lehrkräften Wortschatzübungen durch und stellte sicher, 
dass die Wortschatzplakate aktuell waren und dass die SchülerInnen die Wortschatzmappen einsetzten. Somit konnte sichergestellt werden, dass das Förderprogramm fächerübergreifend eingebunden wurde. Gleichzeitig konnte den Lehrkräften dieses für sie doch recht unbekannte Vorgehen modelliert werden. Schließlich wurde das Wortschatzprogramm (ohne Begleitstudie) vorab mit anderen Lehrkräften in der 5. Jahrgangsstufe im Vorjahr pilotiert, um Erfahrungen zu sammeln und notwendige Änderungen vor der empirischen Phase zu beschließen.

\section{Begleitstudie}

Der Wirksamkeit des in Kap. 3 skizzierten Konzepts wurde in einer quasi-experimentellen Interventionsstudie nachgegangen. Als Untersuchungsdesign wurde ein Prä-/Posttestverfahren mit zwei unabhängigen Gruppen gewählt.

\subsection{Forschungsfrage und Untersuchungshypothesen}

Die Studie geht der Frage nach, inwiefern die unterrichtliche Förderung bildungssprachlichen Wortschatzes SchülerInnen beim Erwerb dieses Wortschatzes unterstützt. Unter Berücksichtigung der in Kap. 3 vorgestellten empirischen Evidenz ergeben sich insgesamt drei Hypothesen:

1. Die am Wortschatztraining teilnehmende Interventionsgruppe verzeichnet im Vergleich zur Kontrollgruppe einen signifikant größeren Zuwachs am getesteten Bildungswortschatz.

2. Die Interventionsgruppe weist gegenüber der Kontrollgruppe insbesondere bei den produktiven Wortschatzaufgaben einen größeren Leistungszuwachs auf, was auf die Erfahrung der Interventionsgruppe mit der Reflexion und der damit einhergehenden Verfestigung neuer Begriffe während der Trainingsphase zurückzuführen ist.

3. Die Interventionsgruppe schneidet im Posttest sowohl bei den trainierten Zieleinheiten als auch bei den nichttrainierten Einheiten besser ab als im Prätest. Grundannahme hierbei ist, dass die Interventionsgruppe eine erhöhte Reflexion von bildungssprachlichen Wortschatzeinheiten trainiert, wodurch sie u.a. auch implizit begegnete Einheiten besser erschließen kann. Es liegt somit ein Transfereffekt vor.

\subsection{Stichprobe}

Insgesamt nahmen 104 FünftklässlerInnen aus vier Klassen eines Gymnasiums (Alter: 10-12 Jahre) an der Studie teil. Die Intervention wurde in einer von der Schulleitung ausgewählten Klasse $(n=23)$ durchgeführt. Überlegungen, das intensiv zu begleitende Training in mehreren Klassen gleichzeitig durchzuführen, wurden nach Absprache mit der Schule aufgrund des personellen Aufwands verworfen. Die anderen drei Klassen dienten als Kontrollgruppe $(n=81)$. Eine im Rahmen eines anderen Projektes ursprünglich geplante Vergleichsintervention mit Fokus auf Leseförderung kam aus schulorganisatorischen Gründen kurzfristig leider nicht zu Stande, sodass in 
der Kontrollgruppe regulärer Deutschunterricht stattfand, bei dem jedoch der gleiche Inhalts- und Lexeminput vorlag (s. 4.3).

In die Datenanalyse wurden nur die Fälle einbezogen, die (1) an sowohl Prä- als auch Posttest teilgenommen haben und (2) mittels eines Anomalieindex ${ }^{1}$ nicht als Ausreißer aufgefallen sind. Insgesamt wurden 94 SchülerInnen (IG: 20; KG: 74) einbezogen. Die Intervention begann zwei Wochen nach Schuljahresanfang und dauerte acht Wochen.

Allen Teilnehmenden gemeinsam war, dass sie gerade den Wechsel auf das Gymnasium vollzogen hatten und diese Schulform sowie die mit ihr einhergehenden Anforderungen neu waren. Die gesamte Stichprobe hatte eine Gymnasialempfehlung von unterschiedlichen Grundschulen erhalten. Das Einzugsgebiet gehört zu den eher sozioökonomisch stärkeren; so sind im betroffenen Stadtteil nur 4,8\% aller Personen unter 15 Jahren SGB-II leistungsberechtigt (städtischer Durchschnitt: 29\%, vgl. SLB 2016). Die Interventions- und Kontrollgruppen unterschieden sich weder signifikant in Bezug auf die Geschlechterverteilung (IG: $43 \%$ w; IG: $45 \%$ w; $\chi^{2}=0,02$, $p=0,89$ ) noch auf die Familiensprache (IG: 1/20 SchülerInnen mit nichtdeutscher Familiensprache; KG: 14/74 SchülerInnen; exakter Test nach Fischer $p=0,18)$. Um diesen letzten Faktor aus theoriebasierten Gründen trotzdem zu kontrollieren, wurde er als Kovariat in die weiteren Analysen aufgenommen; bei keiner dieser Analysen zeigte sich allerdings ein Effekt für die Familiensprache. Die Gruppen unterschieden sich im Prätest (IG: $\bar{x}=48,7, S D=15,0 ; \mathrm{KG}: \bar{x}=52,0, S D=15,9 ; t(92)=0,83$, $p=0,41)$ nicht signifikant. Somit kann davon ausgegangen werden, dass sie sich zu Beginn der Studie in den für die Wortschatzintervention relevanten individuellen Variablen stark ähnelten und sich in Bezug auf den getesteten Wortschatz nicht unterschieden.

\subsection{Systematische Durchführung der Intervention}

$\mathrm{Zu}$ Beginn der achtwöchigen Interventionsphase erhielten die an der Intervention beteiligten Lehrkräfte eine halbtägige Lehrerfortbildung, einen Reader zum Konzept des Wortschatztrainings sowie eine Aktivitätenmappe mit einer Auswahl an Wortschatzübungen. Zusätzlich wurden sie in ihrer ersten Trainingsstunde von einer geschulten Masterstudentin begleitet (s. 3.2) und hatten im Anschluss in einem Einzelgespräch die Möglichkeit, die Stunde zu reflektieren sowie gezielte Nachfragen zu stellen. Der Einbezug der Masterstudentin stellte eine systematische Durchführung des Trainings sowie das Aufgreifen von Lexemen aus verschiedenen Wortschatzbereichen, die im Test (s. 4.4) erhoben wurden, sicher. Darüber hinaus dienten Wortschatzplakat und -mappe als Dokumentationsinstrumente der im Interventionszeitraum behandelten Lexeme. Zusätzlich wurde im Anschluss an das Training eine Befragung der Lehrkräfte durchgeführt, um zu klären, welche Lexeme sie im Rahmen der Intervention eingeführt hatten.

\footnotetext{
1 Es wurden alle SchülerInnen mit einem Anomalieindex > 1,5 ausgeschlossen. Davon waren 7 SchülerInnen aus der Kontrollgruppe und 3 aus der Interventionsgruppe betroffen. Es zeigt sich kein statistischer signifikanter Unterschied zwischen den Fallzahlen an Ausreißern in den beiden Gruppen $\left(\chi^{2}(1)=0,4\right.$; $p=0,69)$.
} 
Während des Interventionszeitraums folgte die Kontrollgruppe dem gleichen Curriculum mit den gleichen Lehrmaterialien wie die Interventionsgruppe. Einige der Lehrkräfte unterrichteten sowohl in Interventions- als auch Kontrollgruppe. Sofern dies der Fall war, wurden die Lehrkräfte explizit dazu angehalten, in der Kontrollgruppe ihren etablierten Unterricht beizubehalten und neue Elemente des Wortschatztrainings erst nach Abschluss des festgesetzten Interventionszeitraums einzubeziehen.

\subsection{Datenerhebung: Erhebungsinstrument und Testitems}

Die Datenerhebung erfolgte mittels eines Wortschatztests, der während des regulären Deutschunterrichts durchgeführt wurde. Weitere qualitative Daten aus den teilnehmenden Beobachtungen (Protokolle), den Lehrerinterviews sowie den Schülerfragebögen werden an dieser Stelle nicht berichtet.

Der Wortschatztest wurde direkt vor der Interventionsphase sowie nach der achtwöchigen Trainingsphase durchgeführt. Prä- und Posttest hatten einen zeitlichen Umfang von jeweils 45 min und bestanden aus neun Aufgaben mit insgesamt 68 Einzelitems. Alle Aufgaben waren an einem von Kurtz (2012a, S. 241) entwickelten Test zur Erfassung des Bildungswortschatzes von Dritt- und Viertklässlern angelehnt. Die Entscheidung für dieses nicht standardisierte Testinstrument liegt erstens darin begründet, dass Testinstrument und Intervention aufeinander abgestimmt sein sollten. Zweitens war es nicht das Ziel der Studie, einen Vergleich mit einer Normierungsstichprobe durchzuführen, sondern gruppenspezifische Unterschiede und konkrete Lerneffekte des Trainings aufzudecken. Ferner wurde entschieden, sowohl kontextfreie, d.h. nicht textuell eingebettete Testung von Einzellexemen, als auch kontexteingebundene Aufgaben in Form von einem Lückentext einzubeziehen, um den differenziellen Effekt der Unterstützung durch Textinferenzen zu berücksichtigen.

Aufgrund eines nachgewiesenen Deckeneffekts wurde die erste, aus Motivationszwecken sehr einfach gestaltete Aufgabe 1a von der Analyse für Hypothesen 1 und 2 ausgeschlossen; sie wird hierfür im Weiteren nicht berücksichtigt. Nach diesem Ausschluss verblieben 61 Items, die sowohl im Prä- als auch im Posttest mit identischen Aufgabenformaten, aber unterschiedlicher Reihenfolge abgefragt wurden. Aus schulorganisatorischen Gründen war es nicht möglich, einen lapsed-posttest anzuschließen. Die Auswahl der im Test erhobenen Wortschatzitems erfolgte über eine Analyse der in allen Fächern verpflichtend einzusetzenden Lehrmaterialien des 5. Jahrgangs, d. h. alle Lernenden begegneten den Lexemen im regulären Unterricht während des Untersuchungszeitraums. Für Hypothese 3 war von Interesse, wie der Trainingsgrad der Lexeme ausfiel. Auf Basis der teilnehmenden Beobachtung und der Lehrerbefragungen konnten 63 Items am Ende der Intervention in drei Kategorien eingeteilt werden: (1) trainiert (19 Lexeme), (2) angesprochen, z. B. von der Lehrkraft kurz mündlich erklärt, aber nicht im Sinne der RVI trainiert (16 Lexeme) und (3) nicht trainiert oder angesprochen, aber Teil des Unterrichtsmaterials (28 Lexeme). Da die Kontrollgruppe nicht begleitet wurde, liegen keine Daten dazu vor, inwieweit bestimmte Items im Regelunterricht angesprochen wurden. 
Zur Beantwortung der zweiten Hypothese wurden die Aufgaben des Prä- und Posttests drei Gruppen zugeordnet: (1) Wiedererkennungsformate zur Prüfung des rezeptiven Wortschatzes, (2) produktive Aufgaben und (3) gemischte Formate.

Bei den (1) dekontextualisierten Wiedererkennungsformaten stand die Bedeutungserkennung im Vordergrund. Hierbei handelte es sich um zwei Aufgaben:

- Antonympaare in zwei Listen zueinander führen (z. B. kommerziell - gemeinnützig)

- Synonympaare in zwei Listen zueinander führen (z. B. ertragreich-fruchtbar)

Die ebenfalls (2) dekontextualisierten, jedoch produktiven Aufgabenformate verlangten dagegen die selbstständige Ergänzung eines (gegensätzlichen) Begriffs oder auch eine alltagssprachliche Erklärung der Bedeutung. In diese Kategorie fielen drei Aufgaben:

- Antonyme aufschreiben (z. B. Was ist das Gegenteil von Zunahme?)

- Synonyme aufschreiben (z.B. Suche ein Wort mit ähnlicher Bedeutung wie „sich etwas einprägen"!)

- Unterschiede zwischen zwei Begriffen erklären (z. B. Rauch-Dampf)

Schließlich sollten (3) gemischte Formate die Unterscheidung sowie die Erarbeitung von Wortschatz im Kontext ermöglichen. Hierbei wurde auf zwei Lückentexte zurückgegriffen:

- Lückentext mit Funktionswörtern vervollständigen (z. B. Löse das Kochsalz destilliertem Wasser von $50^{\circ} \mathrm{C}$.)

- Lückentext mit Inhaltswörtern vervollständigen (z.B. Die Weser kann aus verschiedenen Gründen über das treten.)

Ein letztes Aufgabenformat, das zu den gemischten Aufgaben gerechnet wurde, verlangte, dass SchülerInnen zunächst nicht passende Wörter aus einer Reihe identifizieren und anschließend diese Entscheidung erklären sollten (z.B. Wattenmeer, Deich, Küste, Naturschutzgebiet, Küstenvorland).

\subsection{Datenauswertung}

Die Auswertung der Tests erfolgte unter Einbezug einer im Projekt erstellten Auswertungsleitlinie. Für die Items in jeder Teilaufgabe wurde ein numerisches Ergebnis vergeben, das sich auf die Korrektheit der Antworten bezog. Für jede Teilaufgabe wurde die Summe der Ergebnisse kodiert und anschließend in eine SPSS-Datei überführt.

Anschließend wurde eine explorative Komponentenanalyse ${ }^{2}$ durchgeführt, um zu überprüfen, ob die in Abschn. 4.4 genannte Wortschatzeinteilung (rezeptiver Wortschatz/produktiver Wortschatz/rezeptiv-produktiver Wortschatz) sich durch die Testitems abbildete. Eine Oblimin-Rotation ergab zwei Komponenten. Diese entsprachen jedoch nicht der zunächst zu Grunde gelegten Teilung in produktive, rezeptive und

\footnotetext{
${ }^{2}$ Die Grundvoraussetzungen für die Analyse wurden geprüft und für gegeben befunden: $\mathrm{KMO}=0,75$, signifikanter Bartlett-Test $\left(\chi^{2}(28)=220,24, p=0,00\right)$.
} 
produktiv-rezeptive Aufgabenformate, sondern viel eher der Komplexität der Aufgabe. Während die Items in Faktor 1 (Cronbachs Alpha=0,76) Synonyme, Antonyme oder vereinzelte Wörter im Textzusammenhang vorführen, erfordern die Items in Faktor 2 (Cronbachs Alpha=0,68) entweder die korrekte Auswahl von einzelnen Lexemen aus mehreren, oder sogar eine Begründung der Wahl. Hierbei handelte es sich ausschließlich um die letzte im Abschn. 4.3 genannte Aufgabenkategorie, die zwei Aufgaben enthielt. Diese zwei Itemgruppen werden im Folgenden aus inhaltlichen Gründen ,weniger komplexe Aufgaben“ (Faktor 1) und „,komplexere Aufgaben“ (Faktor 2) genannt.

Als Folge der explorativen Komponentenanalyse und nach interner Diskussion wurde entschieden, die ursprüngliche, für die zweite Hypothese notwendige, jedoch durch die Itemanalyse nicht nachvollziehbare Trennung in drei Aufgabenformate aufzugeben und stattdessen die beiden aus den Daten extrahierten Faktoren gesondert zu analysieren. Obwohl der Alpha-Wert des zweiten Faktors unter der empfohlenen 0,7-Grenze lag, wurde er beibehalten, um einem Informationsverlust entgegenzuwirken (s. Schmitt 1996, S. 351 f.).

\section{Ergebnisse}

\subsection{Gesamteffekte auf den getesteten Bildungswortschatz}

Zur Prüfung des Gesamteffektes auf den getesteten Bildungswortschatz wurde zunächst eine ANOVA mit Messwiederholung an dem Summenwert aller Aufgaben (außer Aufgabe 1a, s. 4.4) durchgeführt.

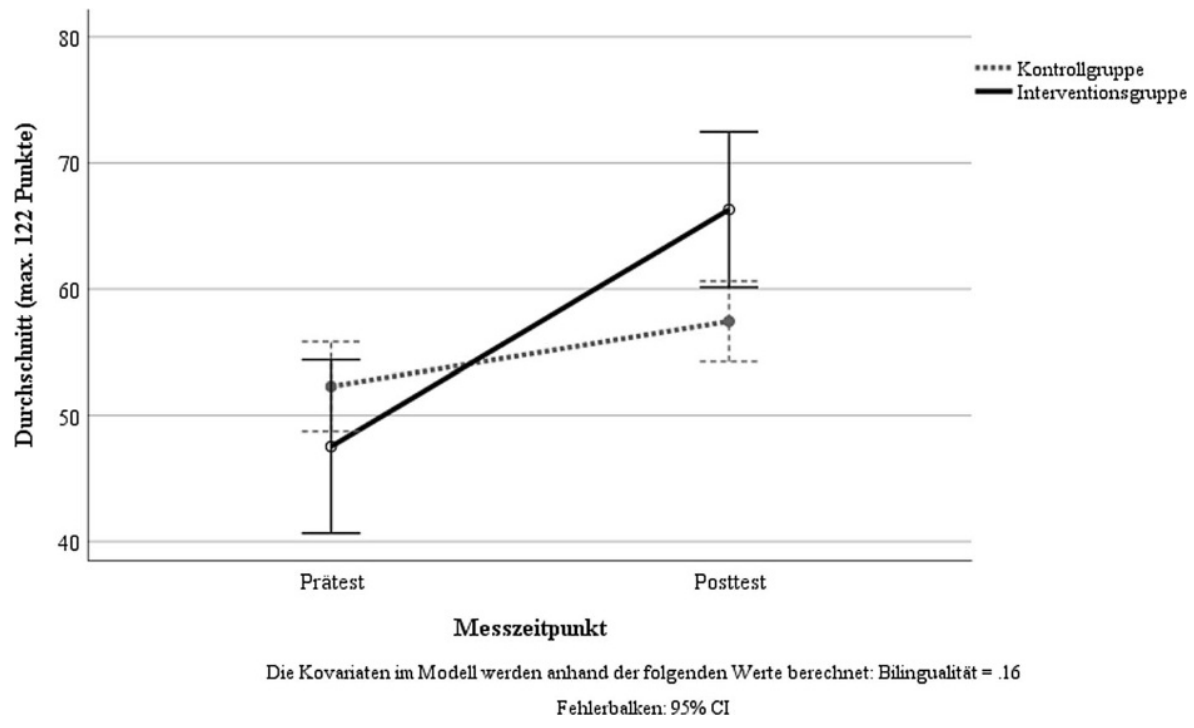

Abb. 1 Ergebnisse der Interventions- und Kontrollgruppen im Prä- und Posttest 
Im Prätest ergaben sich keine Unterschiede zwischen den Gruppen (s. 4.2). Im Posttest waren dennoch Differenzen zu verzeichnen (IG: $\bar{x}=67,3, S D=14,6$; KG: $\bar{x}=57,2, S D=13,9$ ). Diese wurden auch durch die ANOVA bestätigt. Obwohl die Gruppe sich insgesamt verbesserte (Haupteffekt Messzeitpunkt: $F(1,92)=110,2$, $\left.p=0,000,{ }_{p} \eta^{2}=0,55\right)$, war auch die Interaktion zwischen Messzeitpunkt und Gruppe signifikant mit großer Effektstärke $\left(F(1,92)=39,5, p=0,000,{ }_{p} \eta^{2}=0,30\right)$. Das bedeutet, dass die Interventionsgruppe sich stärker als die Kontrollgruppe verbesserte. Die erste Hypothese lässt sich somit bestätigen. Abb. 1 zeigt den Vergleich der beiden Gruppen im Prä- und Posttest.

\subsection{Ergebnisse der produktiven und rezeptiven Wortschatzaufgaben}

Die zweite Hypothese erwies sich als nicht testbar. Eine explorative Komponentenanalyse (s. 4.5) zeigte, dass die theoretische Vorannahme, die Aufgaben seien in rezeptiv vs. produktiv klassifizierbar, nicht zutraf. Stattdessen zeichneten sich zwei Aufgabengruppen ab, die sich hinsichtlich ihrer Komplexität unterschieden. Die Ergebnisse auf Basis der angepassten Aufgabengruppen werden im folgenden Unterkapitel beschrieben.

\subsection{Ergebnisse der Wortschatzsaufgaben nach Grad ihrer Komplexität}

Die zwei Itemgruppen komplexere Aufgaben und weniger komplexe Aufgaben werden separat analysiert (s. 4.5), um zu bestimmen, ob Interventions- und Kontrollgruppen differenzielle Änderungen erfahren. Es handelt sich hierbei nicht um eine hypothesenprüfende Analyse, vielmehr sollte hypothesenentwickelnd, d.h. explorativ vorgegangen werden. Hierfür wurden ANOVAs mit Messwiederholung eingesetzt, wobei aufgrund der wiederholten Analyse bereits analysierter Daten eine Bonferroni-Korrektur durchgeführt wurde. Im Folgenden werden nur die Ergebnisse der Interaktion zwischen Messzeitpunkt und Gruppe berichtet, da nur diese zur Prüfung der Intervention relevant sind.

Für die weniger komplexen Aufgaben ergaben sich, wie zu erwarten, keine Unterschiede zwischen beiden Gruppen beim Prätest (IG: $\bar{x}=36,5, \mathrm{SD}=15,2$; KG: $\bar{x}=41,1, \mathrm{SD}=13,2 ; t(92)=1,03, p=0,31)$. Beim Posttest erzielte die Interventionsgruppe $(\bar{x}=54,1, S D=12,3)$ höhere Ergebnisse als die Kontrollgruppe $(\bar{x}=46,4$, $S D=11,2)$. Eine ANOVA zeigte einen signifikanten und großen Haupteffekt für die Interaktion zwischen Messzeitpunkt und Gruppe, d.h. die Interventionsgruppe verbesserte sich stärker als die Kontrollgruppe $\left(F(1,92)=29,7, p=0,000,{ }_{p} \eta^{2}=0,25\right)$. Abb. 2 zeigt den Vergleich der beiden Gruppe für diesen Faktor.

Auch für die komplexeren Aufgaben waren Unterschiede erst nach dem Prätest (IG: $\bar{x}=9,6, S D=3,8 ; \mathrm{KG}: \bar{x}=10,9, S D=4,8 ; t(92)=1,1, p=0,06) \mathrm{zu}$ verzeichnen. So zeigte sich ein signifikanter, mittlerer Haupteffekt für die Interaktion zwischen Messzeitpunkt und Gruppe $\left(F(1,92)=11,4, p=0,001,{ }_{p} \eta^{2}=0,11\right)$. Das bedeutet, dass sich die Interventionsgruppe auch für diesen Aufgabentypus im Laufe der Zeit stärker verbesserte als die Kontrollgruppe (Abb. 3). 


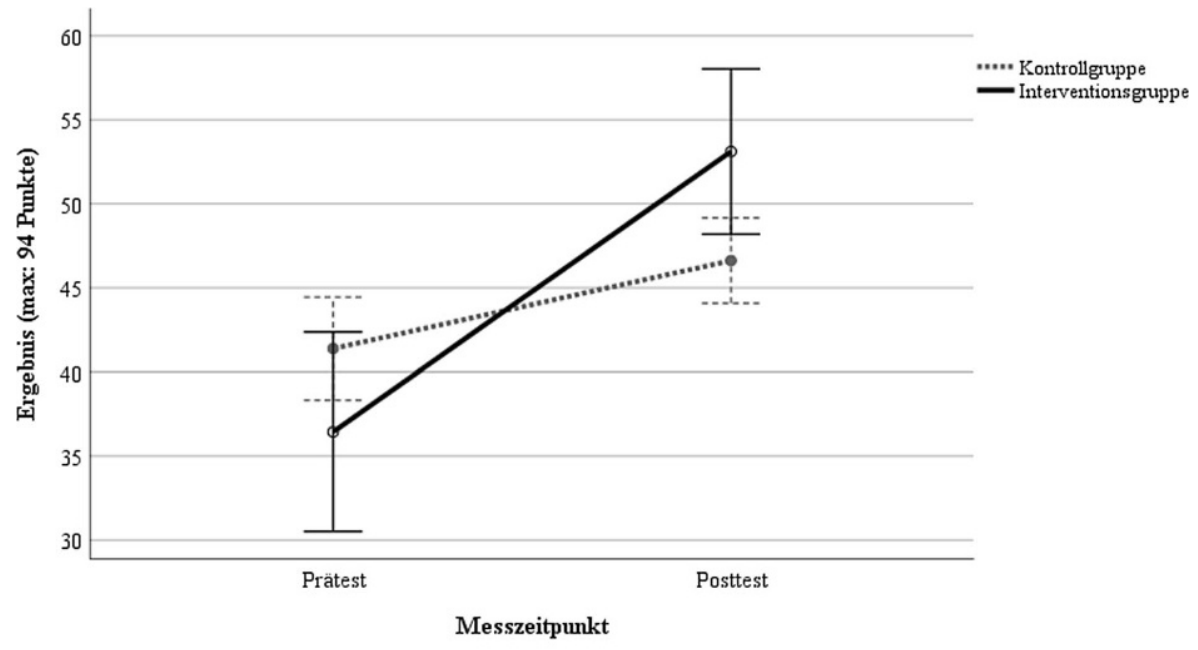

Die Kovariaten in Modell werden anhand der folgenden Werte berechnet: Bilingualität $=.16$ Fehlerbalken: $95 \% \mathrm{CI}$

Abb. 2 Ergebnisse der Interventions- und Kontrollgruppen im Prä- und Posttest für weniger komplexe Aufgaben

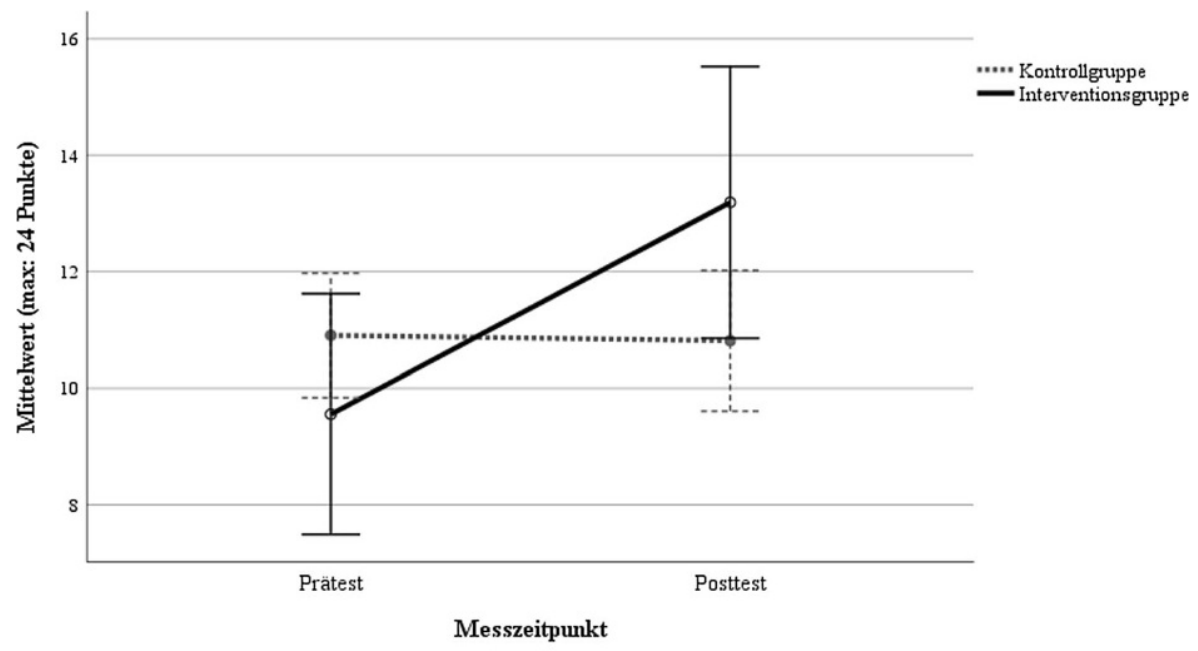

Die Kovariaten in Modell werden anhand der folgenden Werte berechnet: Bilingualität $=.16$ Fehlerbalken: $95 \% \mathrm{CI}$

Abb. 3 Ergebnisse der Interventions- und Kontrollgruppen im Prä- und Posttest für komplexere Aufgaben

\subsection{Effekte expliziten Trainings bei der Interventionsgruppe}

Von weiterem Interesse war, ob die Interventionsgruppe sich v.a. bei den explizit trainierten Lexemen verbesserte, oder ob die Entwicklung auch bei nichttrainierten Wortschatzitems zu verzeichnen war, also ob das Training trotz seiner kurzen Lauf- 


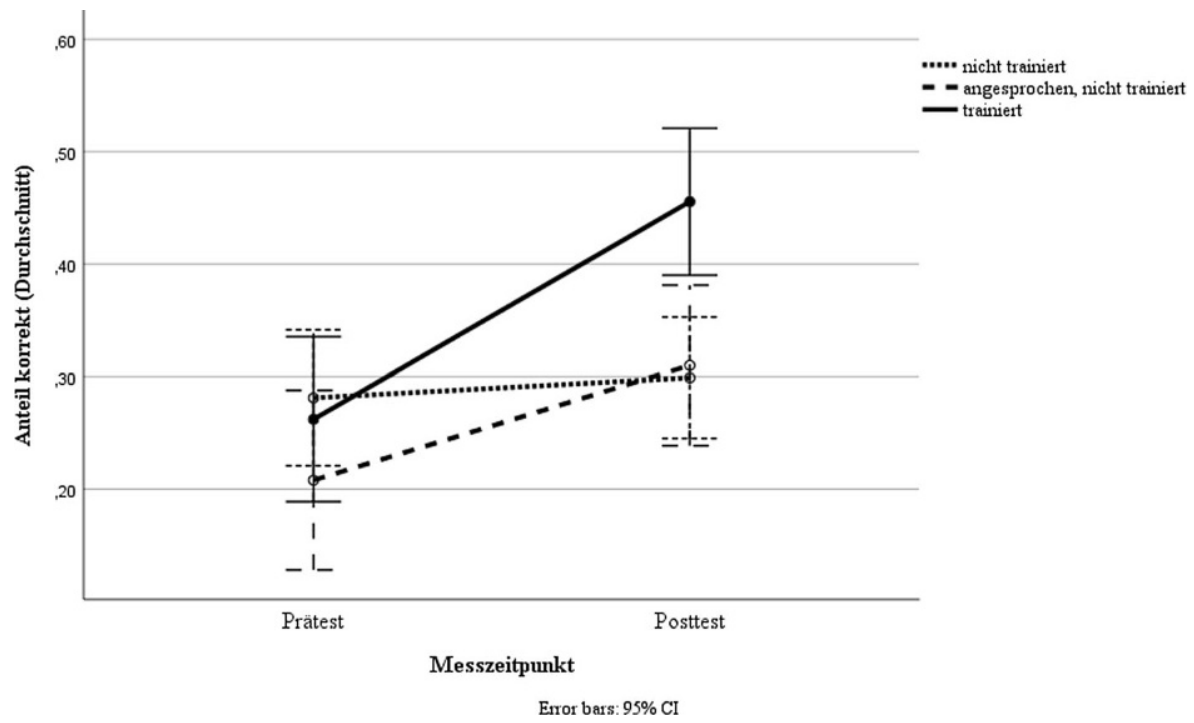

Abb. 4 Durchschnittlicher Anteil korrekter Items nach Trainingsgrad beim Prä- und Posttest

zeit auch zu einem vertieften Verständnis von unbehandelten Vokabeln und somit zu einem Transfereffekt führte. Hierfür wurden die Wortschatzitems in drei Gruppen eingeteilt: (1) trainiert, (2) angesprochen, aber nicht trainiert und (3) nicht trainiert bzw. besprochen (aber immer noch Teil des Unterrichtsmaterials) (s. 4.4). Bei der folgenden Darstellung ist zu beachten, dass aufgrund der unterschiedlichen maximalen Punktwerte für die unterschiedlichen Gruppen die Mittelwerte nur innerhalb der Lexemgruppen vergleichbar sind. Der Vergleichbarkeit halber werden die Ergebnisse in der grafischen Darstellung deswegen prozentual anstatt anhand von Rohwerten präsentiert.

Eine ANOVA mit post-hoc t-Tests ergab einen signifikanten und großen Haupteffekt für die Interaktion von Trainingsgrad und Messzeitpunkt $(F(62,2)=23,3$, $\left.p=0,000,{ }_{p} \eta^{2}=0,44\right)$. Obwohl sich die Lernenden bei den nicht behandelten Lexemen vom Prätest $(\bar{x}=12,8, S D=8,3)$ zum Posttest $(\bar{x}=13,6, S D=8,3)$ offenbar nicht verbesserten $(t(27)=1,3, p=0,21)$, zeichnete sich eine positive Entwicklung bei den angesprochenen Items (Prätest: $\bar{x}=9,5, S D=6,1$; Posttest: $\bar{x}=14,1, S D=5,9$; $t(15)=7,0, p=0,000)$ und bei den trainierten Items (Prätest: $\bar{x}=11,9, S D=6,6$; Posttest: $\bar{x}=20,7, S D=7,2 ; t(18)=7,2, p=0,000)$ ab (Abb. 4). Das bedeutet, dass die SchülerInnen der Interventionsgruppe tatsächlich unterschiedlichen Erfolg zeigten, je nachdem, ob die getesteten Wortschatzeinheiten explizit trainiert, nur angesprochen, oder nicht behandelt wurden. Die nachgewiesene Verbesserung im Wortschatz war also nicht auf eine allgemeine Entwicklung, sondern dezidiert auf die Intervention zurückzuführen.

Trotz der signifikanten Verbesserung muss hier vermerkt werden, dass die SchülerInnen selbst bei den trainierten Lexemen im Durchschnitt weniger als die Hälfte der möglichen Punkte beim Posttest erreichten. 


\section{Diskussion und Ausblick}

Im Rahmen der Interventionsstudie war von Interesse, ob SchülerInnen, die an einem integrativen Wortschatztraining auf Basis der RVI nach Beck et al. (2002) über acht Wochen teilnahmen, im Vergleich zu einer Kontrollgruppe einen Wortschatzzuwachs aufzeigten. Die Grundlage für die Intervention bildete ein Pool von im Regelunterricht relevanten Einzellexemen und Wortgruppen, die in der Interventionsgruppe entweder trainiert, nur angesprochen oder nicht explizit behandelt wurden, aber Teil des Unterrichtsmaterials waren. In Interventions- sowie Kontrollgruppe waren alle Lexeme Bestandteil der regulären Unterrichtsmaterialien.

Annahme der ersten Hypothese war, dass die am Wortschatztraining teilnehmende Interventionsgruppe im Vergleich zur Kontrollgruppe einen signifikant größeren Zuwachs am getesteten Bildungswortschatz aufweisen würde. Dies konnte bestätigt werden: Obwohl beide Gruppen sich im Laufe der Untersuchung verbesserten, verzeichnete die Interventionsgruppe einen deutlich stärkeren Zuwachs als die Kontrollgruppe.

Die Frage, ob diese Differenzen an der Art der Wortschatzaufgabe lagen, sollte durch die Untersuchung der zweiten Hypothese geprüft werden. Hierfür wurden die Wortschatzaufgaben in produktive und rezeptive Aufgaben unterteilt. Allerdings zeigte eine explorative Komponentenanalyse, dass sich diese theoriegeleitete Einteilung nicht in den Daten widerspiegelte. Stattdessen ergaben sich zwei Aufgabengruppen, die sich bzgl. ihres Grads an Komplexität unterschieden. Beide Gruppen haben sich bei den zwei Aufgabentypen verbessert, aber die Interventionsgruppe zeigte auch hier signifikant bessere Ergebnisse als die Kontrollgruppe. Dies deutet darauf hin, dass das Training auch in anspruchsvolleren Kontexten unterstützend wirkt: in diesem Fall bei Aufgaben, bei denen nicht nur zwei semantische Einheiten in Verbindung gebracht und/oder erklärt werden müssen, sondern mehrere Lexeme gleichzeitig gegenübergestellt und ihre Unterschiede bzw. Gemeinsamkeiten beschrieben werden sollen.

Schließlich war von Interesse, ob ein Transfereffekt auftritt, d.h., ob sich die Interventionsgruppe nur bei den explizit trainierten Lexemen verbessert oder ob eine vermutete erhöhte Reflexion von bildungssprachlichen Wortschatzeinheiten zu einem Transfereffekt auch in Bezug auf weitere nicht explizit trainierte Lexeme führt. Es zeigte sich, dass Wortschatzeinheiten, die zwar im Unterricht vorkamen, aber weder angesprochen noch ausdrücklich trainiert wurden, im Posttest nicht besser bearbeitet wurden als im Prätest. Anders war es bei den weiteren zwei Wortschatzgruppen: Wurde ein Lexem trainiert oder zumindest angesprochen, konnten SchülerInnen die entsprechenden Aufgaben beim Posttest besser lösen. Dies bestätigt die Effektivität der Wortschatzübungen - und zeigt zudem, dass die Effekte sich nicht unmittelbar auf andere Bereiche transferieren lassen. Ein rein implizites Wortschatzlernen im Sinne einer rezeptiven Begegnung erwies sich somit nicht als erfolgreich. Stattdessen bedarf es offenbar einer dezidierten Bewusstmachung relevanter Wortschatzeinheiten, um den FünftklässlerInnen den notwendigen Anstoß zum Merken und Verfestigen zu geben.

Insgesamt konnten positive Effekte des Förderkonzepts in einem relativ kurzen Zeitraum von nur acht Wochen nachgewiesen werden. Diese Ergebnisse entsprechen 
denen von Kurtz (2012c), wenn aus der von ihr untersuchten Grundschulstichprobe nur die Klassen mit geschulten Förderlehrkräften einbezogen werden: Nach einem Jahr RVI hatten 3. und 4. Klassen mit erfahrenen und methodisch unterstützten Lehrkräften signifikant bessere Ergebnisse erzielt als die Kontrollklassen sowie als die Klassen mit unerfahrenen Lehrkräften. Dies zeigt, dass die Intervention auch für ältere SchülerInnen in Deutschland effektiv war. Ebenfalls stimmen die Ergebnisse mit denen aus dem angloamerikanischen Raum überein (s.u.v.a. Beck et al. 2008; zuletzt McKeown et al. 2018), die sowohl den Vorteil eines integrativen Wortschatztrainings als auch die Notwendigkeit der intensiven Unterstützung von v.a. unerfahrenen Lehrkräften betonen.

Auch in der vorliegenden Untersuchung scheint ein wesentlicher Faktor für den Erfolg der Maßnahme die Bereitschaft und Motivation sowie die begleitende Unterstützung der involvierten Lehrkräfte gewesen zu sein, wie die (hier nicht berichteten) Ergebnisse der teilnehmenden Beobachtung zeigten. Aber auch bei positiver Aufnahme des Konzepts fiel das Engagement unterschiedlich stark aus. Einige der Lehrkräfte bereiteten aktiv Wortschatzmaterialien vor und suchten gezielt zu trainierende Lexeme aus, wohingegen andere zwar gelegentlich Lexeme im Unterricht aufgriffen, diese jedoch nur selten in Übungen vertieften oder in der Wortschatzmappe notierten. Dies zeigte sich auch bei der fehlenden Routine im Einsatz der Wortschatzmappen. Gerne einbezogen wurden dagegen die Wortschatzplakate, die nach einer etwa zweiwöchigen Anlaufphase von allen Lehrkräften in den Unterricht aufgenommen wurden.

Als problematisch hat sich zudem die Zuständigkeit für organisatorische und fachübergreifende Aspekte des Wortschatztrainings (Wechsel der Plakate, Bereitstellung von Unterrichtszeit als Übungszeit) erwiesen. Es entstand der Eindruck, ein Teil der Lehrkräfte sehe sich vorrangig als Fach- und nicht als Sprachlehrkraft und fühle sich somit für die Wortschatzerweiterung weniger verantwortlich. Die Unterstützung der Masterstudentin hat in diesem Sinne maßgeblich zum Erfolg des Projektes innerhalb des Projektzeitraums beigetragen.

Insgesamt wurde deutlich, dass das Förderkonzept, wie all solche Konzepte, nicht nur die Motivation und Kooperation aller involvierten Beteiligten, sondern auch eine gewisse Routine voraussetzt. An der Kooperationsschule erweist sich diese in mehreren Klassen inzwischen als gegeben und die Maßnahme wird mit relativ wenig Aufwand und mit nur noch sporadischer studentischer Unterstützung funktional in den Regelunterricht integriert.

\section{Mögliche Einschränkungen der Studie}

Da die SchülerInnen im Klassenverbund blieben, konnte keine randomisierte Zuteilung zu Interventions- und Kontrollgruppe erfolgen, sodass klassenspezifische Effekte nicht ausgeschlossen werden können. Ebenso ist nicht auszuschließen, dass die Zuschreibung als Interventionsgruppe oder das Engagement der jeweils unterrichtenden Lehrkräfte das Ergebnis der Studie beeinflusste. Zur Steigerung der empirischen Evidenz wäre es zudem sinnvoll, eine weitere Interventionsgruppe einzubeziehen, die an einem anderen Interventionsverfahren teilnimmt. 
Eine erneute größer angelegte Erhebung zur Validierung der Ergebnisse der explorativen Studie sollte eine Kontrolle der genannten Einflussfaktoren beinhalten. Darüber hinaus wäre eine systematische Kontrolle der in Interventionsgruppe und Kontrollgruppe behandelten Wortschatzeinheiten sowie deren Umfang anzustreben, die über eine Befragung der unterrichtenden Lehrkräfte und die Analyse der Wortschatzplakate hinausgeht. Auch wären die eingesetzten Wortschatztests systematisch zu validieren.

Funding Open Access funding provided by Projekt DEAL.

Open Access Dieser Artikel wird unter der Creative Commons Namensnennung 4.0 International Lizenz veröffentlicht, welche die Nutzung, Vervielfältigung, Bearbeitung, Verbreitung und Wiedergabe in jeglichem Medium und Format erlaubt, sofern Sie den/die ursprünglichen Autor(en) und die Quelle ordnungsgemäß nennen, einen Link zur Creative Commons Lizenz beifügen und angeben, ob Änderungen vorgenommen wurden.

Die in diesem Artikel enthaltenen Bilder und sonstiges Drittmaterial unterliegen ebenfalls der genannten Creative Commons Lizenz, sofern sich aus der Abbildungslegende nichts anderes ergibt. Sofern das betreffende Material nicht unter der genannten Creative Commons Lizenz steht und die betreffende Handlung nicht nach gesetzlichen Vorschriften erlaubt ist, ist für die oben aufgeführten Weiterverwendungen des Materials die Einwilligung des jeweiligen Rechteinhabers einzuholen.

Weitere Details zur Lizenz entnehmen Sie bitte der Lizenzinformation auf http://creativecommons.org/ licenses/by/4.0/deed.de.

\section{Literatur}

Ahrenholz, B. (2010). Bildungssprache im Sachunterricht der Grundschule. In B. Ahrenholz (Hrsg.), Fachunterricht und Deutsch als Zweitsprache (Bd. 2, S. 15-36). Tübingen: Narr Francke Attempto.

Beck, I., McKeown, M., \& Kucan, L. (2002). Bringing words to life - robust vocabulary instruction. New York: Guilford.

Beck, I., McKeown, M., \& Kucan, L. (2008). Creating robust vocabulary - frequently asked questions and extended examples. New York: Guilford.

Beck, I., Perfetti, C., \& McKeown, M. (1982). Effects of long-term vocabulary instruction on lexical access and reading comprehension. Journal of Educational Psychology, 74, 506-521.

Behrens, H. (2009). Konstruktionen im Spracherwerb. Zeitschrift für Germanistische Linguistik, 37(3), $427-444$.

Feilke, H. (2012). Bildungssprachliche Kompetenzen - fördern und entwickeln. Praxis Deutsch, 233, 4-13.

Gogolin, I. (2006). Bilingualität und die Bildungssprache der Schule. In P. Mecheril \& T. Quehl (Hrsg.), Die Macht der Sprachen. Englische Perspektiven auf die mehrsprachige Schule (S. 79-85). Münster: Waxmann.

Gogolin, I., Neumann, A., \& Roth, H.-J. (2007). Schulversuch bilinguale Grundschulklassen in Hamburg - Wissenschaftliche Begleitung. Abschlussbericht über die italienisch-deutschen, portugiesisch-deutschen und spanisch-deutschen Modellklassen. Hamburg: Universität zu Köln/Universität Hamburg.

Heppt, B. (2016). Verständnis von Bildungssprache bei Kindern mit deutscher und nicht-deutscher Familiensprache (Dissertationsschrift). Berlin: Humboldt-Universität, Lebenswissenschaftliche Fakultät.

Koch, P., \& Österreicher, W. (1985). Sprache der Nähe - Sprache der Distanz. Mündlichkeit und Schriftlichkeit im Spannungsfeld von Sprachtheorie und Sprachgeschichte. Romanistisches Jahrbuch, 36, $15-43$.

Kurtz, G. (2012a). Bildungssprache - Sprachbildung: Leistungen in verschiedenen Wortschatzgebieten bei Dritt- und Viertklässlern mit Deutsch als Erst- und Zweitsprache. In B. Ahrenholz \& W. Knapp (Hrsg.), Sprachstand erheben - Spracherwerb erforschen. 2010 (Beiträge aus dem 6. Workshop „Kinder mit Migrationshintergrund“", S. 241-261). Stuttgart: Klett.

Kurtz, G. (2012b). Bildungswortschatz trainieren - Robusten Wortschatz aufbauen. In S. Merten \& K. Kuhs (Hrsg.), Perspektiven empirischer Sprachdidaktik (S. 71-90). Trier: WVT. 
Kurtz, G. (2012c). Effekte der integrierten Sprachförderung in 3. und 4. Klassen auf Leistungen in einem Wortschatztest. In S. Merten \& K. Kuhs (Hrsg.), Perspektiven empirischer Sprachdidaktik (S. 179-193). Trier: WVT.

Marx, N. (2018). Förderung, aber welchen Inhalts? Didaktische Perspektiven auf Bildungssprache(n) im DaF-Unterricht. Info DaF, 45(4), 401-422.

Mckee, S. (2012). Reading comprehension, what we know: a review of research 1995 to 2011. Language Testing in Asia, 2, 1-14.

McKeown, M., Beck, I., Omanson, R., \& Pople, M. (1985). Some effects of the nature and frequency of vocabulary instruction on the knowledge and use of words. Reading Research Quarterly, 20, 522-535.

McKeown, M., Crosson, A., Moore, D., \& Beck, I. (2018). Word knowledge and comprehension effects of an academic vocabulary intervention for middle school students. American Educational Research Journal, 55(3), 572-616.

Morek, M., \& Heller, V. (2012). Bildungssprache - Kommunikative, epistemische, soziale und interaktive Aspekte ihres Gebrauchs. Zeitschrift für angewandte Linguistik, 57(1), 67-101.

Ortner, H. (2009). Rhetorisch-stilistische Eigenschaften der Bildungssprache. In U. Fix, A. Gardt \& J. Knape (Hrsg.), Rhetorik und Stilistik (Bd. 2, S. 2227-2240). Berlin: De Gruyter.

Pigada, M., \& Schmitt, N. (2006). Vocabulary acquisition from extensive reading. A case study. Reading in a Foreign Language, 18, 1-28.

Quasthoff, U. (2009). Entwicklung der mündlichen Kommunikationskompetenz. In M. Becker-Mrotzek (Hrsg.), Mündliche Kommunikation und Gesprächsdidaktik (Teilband Mündlichkeit in der Reihe „Deutschunterricht in Theorie und Praxis“, S. 84-100). Baltmannsweiler: Schneider Hohengehren.

Schleppegrell, M. (2012). Academic language in teaching and learning: introduction to the special issue. The Elementary School Journal, 112(3), 409-418.

Schmitt, N. (1996). Uses and abuses of coefficient alpha. Psychological Assessment, 8(4), 350-353.

SLB = Statistisches Landesamt Bremen (2016). Bremer Ortsteilatlas. http://www.statistik-bremen.de/ tabellen/kleinraum/ortsteilatlas/atlas.html. Zugegriffen: 12. Okt. 2018.

Snow, C., \& Uccelli, P. (2009). The challenge of academic language. In N. Torrance \& D. Olson (Hrsg.), The cambridge handbook of literacy (S. 112-133). Cambridge: Cambridge University Press.

Stahns, R. (2016). Bildungssprachliche Merkmale von Texten und Items. Zur Operationalisierung des Konstrukts „Bildungssprache“. Didaktik Deutsch, 41, 44-55.

Steinhoff, T. (2019). Konzeptualisierung bildungssprachlicher Kompetenzen. Anregungen aus der pragmatischen und funktionalen Linguistik und Sprachdidaktik. Zeitschrift für Angewandte Linguistik, 71(1), 327-352.

Steinhoff, T., Borgmeier, H., Brosowski, T., \& Marx, N. (2019). Förderung des mündlichen Gebrauchs der Bildungslexik in den Sachfächern der Sekundarstufe I. In C. Titz, S. Weber, H. Wagner, A. Ropeter, S. Geyer \& M. Hasselhorn (Hrsg.), Sprach- und Schriftsprachförderung wirksam gestalten: Innovative Konzepte und Forschungsimpulse (S. 135-155). Stuttgart: Kohlhammer.

Steinhoff, T. (2009). Wortschatz - eine Schaltstelle für den schulischen Spracherwerb. Siegener Papiere zur Aneignung sprachlicher Strukturformen (SPASS) 17. http://www.studiger.tu-dortmund.de/images/ Steinhoff_Wortschatz_SPAsS_2009.pdf. Zugegriffen: 12. Okt. 2018.

Steinig, W. (2016). Sprache, Bildung und soziale Herkunft. In J. Kilian, B. Brouër \& D. Lüttenberg (Hrsg.), Handbuch Sprache in der Bildung (S. 68-98). Berlin: De Gruyter Mouton.

Townsend, D., Filppini, A., Collins, P., \& Biancarosa, G. (2012). Evidence for the importance of academic word knowledge for the academic achievement of diverse middle school students. The Elementary School Journal, 112(3), 497-518.

Tschirner, E. (2010). Wortschatz. In H. J. Krumm, C. Fandrych, B. Hufeisen \& C. Riemer (Hrsg.), Deutsch als Fremd- und Zweitsprache. Ein internationales Handbuch (S. 236-245). Berlin: De Gruyter Mouton.

Ulrich, W. (2011). Wortschatzarbeit im Deutschunterricht. ide - Zeitschrift für den Deutschunterricht in Wissenschaft und Schule, 35(1), 9-21.

Willenberg, H. (2008). Wortschatz Deutsch. In DESI-Konsortium (Hrsg.), Unterricht und Kompetenzerwerb in Deutsch und Englisch - Ergebnisse der DESI-Studie (S. 71-80). Weinheim: Beltz. 